\title{
LA AVENIDA DE LA LUZ, UNA CALLE SUBTERRÁNEA EN BARCELONA
}

POR

\author{
MARÍA XALABARDER AULET
}

Inaugurada en 1940, la Avenida de la Luz fue una de las primeras galerías comerciales modernas de Barcelona, y la primera subterránea de Europa. Constituyó uno de los espacios urbanos más atractivos y destacados de la Barcelona de la postguerra española, siendo un ejemplo que siguieron más tarde otras ciudades europeas. Sita bajo la calle Pelayo, entre la Plaza de Cataluña y la confluencia con la calle Balmes y Vergara, ocupaba el vestíbulo de acceso a la estación de Plaza de Cataluña de los Ferrocarriles de Barcelona a Sarriá - hoy Ferrocarriles Catalanes de la Generalitat-, en pleno centro de la ciudad. Sobre la Avenida de la Luz se hallaba un solar que llegó a ser conocido popularmente como triángulo de la vergüenza, por el fracaso de todos los proyectos que trataron de urbanizar una área tan céntrica y simbólica de la ciudad. El proyecto constructivo del centro comercial El Triangle, ha transformado finalmente aquel solar en un triángulo de oro. Las obras de construcción del nuevo complejo obligaron a cerrar la Avenida de la Luz, que permaneció a oscuras tras una tapia desde 1990 hasta el 11 de noviembre de 1998, día en el cual se inauguró el nuevo edificio de la Plaza de Cataluña. El espacio subterráneo que ocupaba la Avenida de la Luz, ha sido parcialmente ocupado por la firma francesa Sephora, dedicada a la venta de perfumería y cosméticos.

Este artículo pretende dar cuenta de los procesos de formación y desarrollo de este singular espacio urbano y de consumo, y de las circunstancias y agentes urbanos que participaron en él. También, y desde

Estudios Geográficos

Tomo LX, n. ${ }^{\circ} 236$, julio-septiembre 1999 
una perspectiva urbanística, dedicamos un episodio a la Ciudad Subterránea de la Luz, en tanto que proyecto de gran envergadura que debía secundar la apertura de la Avenida de la Luz y que constituía el objetivo último de su promotor. De este modo, el estudio se refiere a un espacio que ha ocupado un lugar destacado en el paisaje urbano barcelonés y en la memoria histórica de la ciudad.

La investigación plantea también el tema de la relación entre proyectos urbanísticos y las estrategias de las grandes compañías de electricidad, en tanto que potentes grupos empresariales preocupados por incrementar el consumo y la demanda de esta energía, especialmente en el ámbito doméstico y particular a través de la introducción de nuevos productos electrodomésticos, y participando en la creación de nuevos espacios de consumo. No podemos olvidar que la compañía Ferrocarriles de Sarriá formaba parte de la Barcelona Traction, principal grupo de electricidad en Cataluña en el momento de inauguración de la Avenida de la Luz. Esta firma actuaba en Barcelona a través de la Compañía Barcelonesa de Electricidad, desarrollando una agresiva política de ventas que encontraba en los comercios y servicios un mercado potencial muy rentable según datos de 1935, éste sector consumía alrededor de un $40 \%$ de la luz eléctrica producida ${ }^{1}$.

La documentación aquí utilizada se basa en los archivos públicos y privados, en un abundante material de crónicas periodísticas, en entrevistas en profundidad a personas directamente implicadas en la Avenida, y en las escasas referencias bibliográficas que hemos podido encontrar. Con todo, este estudio se ha visto limitado por hallarse destruida gran parte de la documentación archivística de la Avenida de la Luz, S.L., empresa gestora de las galerías comerciales. Además, y debido a los impedimentos que ha impuesto la compañía, no hemos podido consultar los archivos de Ferrocarriles Catalanes de la Generalitat en relación a la Avenida de la Luz.

La Avenida de la Luz fue un exponente claro de modernidad para la Barcelona de mediados del siglo xx. Ello se debía tanto a la función que desarrollaba, por la conjugación moderna de usos comerciales y prestación de servicios diversos, como por la estética de raíces futuristas, y

${ }^{1}$ Urteaga, L.: «El consumo de Electricidad en Barcelona, 1897-1935». In Capel, H. y Linteau, P. (coord.), Barcelona Montréal: desarrollo urbano comparado, Barcelona, 1998, Universidad de Barcelona, pp. 259-283. Véase también Trédé, M. (coord.): Électricité et Électrification dans le monde, 1880-1980, París, 1990, ELEC, p. 561. 
el empleo de la energía eléctrica como máximo exponente y símbolo de progreso. Cabe pues, a modo de contextualización, hablar de los procesos económicos y culturales que actuaban en el marco histórico y que hicieron posible el que la iniciativa privada, alentada por una política de reconstrucción postbélica y de propagandismo fatuo del régimen, transformase un espacio aparentemente anodino en uno de los más destacados de la ciudad de postguerra.

Impacto socio-cultural y económico de la electrificación de Barcelona

Barcelona recibió la llegada del siglo xx en un ambiente intelectual y cultural preocupado por la aplicación de nuevas tecnologías en la industria y el progreso económico. La producción y extensión de la electricidad, como nueva y potente fuente energética tanto para consumo industrial como particular, supuso un factor fundamental en el desarrollo industrial y urbano que vivió la ciudad. Una vez ganada la batalla del alumbrado público frente al gas, asegurada la de los tranvías, y abierta la del suministro energético a la industria, las compañías eléctricas que operaban en la ciudad se dirigieron hacia el mercado doméstico, que aparecía como el nuevo y gran frente de expansión de la demanda. Así, en los años 1920 y, sobre todo en 1930 estas compañías emprendieron importantes campañas para difundir el consumo de electricidad en el hogar, a través de anuncios en la prensa, en los teatros y cines, charlas radiofónicas así como todo tipo de recursos publicitarios. Por otra parte, comenzaba la comercialización masiva de aparatos eléctricos de uso doméstico, como la radio y el secador de pelo, a los que siguen el aspirador y el frigorífico ${ }^{2}$.

Paralelamente, la electricidad ya venía desempeñando, también, un importante papel en el desarrollo cultural y estético de la primera mitad del siglo xx. Desde muy pronto, el desarrollo técnico y las innovaciones aplicadas a la industria eléctrica eran alentadas e impulsadas por una so-

${ }^{2}$ Capel, H.: «La electricidad en Cataluña, una historia por hacer Conclusiones». In Las Tres Chimeneas implantación industrial, cambio tecnológico y transformación de un espacio urbano barcelonés, Barcelona, FECSA, 1994, 3 vols. Véase también: NYE, D.: Electrifying America Social Meanings of a New Technology 1880-1940, London, MIT press, 1992 , p. 479. 
ciedad dinámica y consciente de la necesidad de apostar por la ciencia y la formación. Ejemplo de ello son, además de la universidad, la existencia de la Academia de las Ciencias, la Escuela de Ingenieros Industriales, o los centros para la formación de numerosos instaladores, montadores y obreros especializados. Todo ello permitió una rápida difusión de los conocimientos sobre la electricidad. Las nuevas capacidades técnicas y tecnológicas permitían usos y aplicaciones antes insospechados. La electricidad iluminaba las estancias modernas de la vida cotidiana, y proporcionaba un nuevo paisaje urbano espectacular.

En este marco, no es extraño que ya en 1913 los industriales y políticos catalanes se plantearan organizar una Exposición Internacional de Industrias Eléctricas, que hubiese sido el primer salón monográfico de estas características en España. Aunque la iniciativa no llegó a cuajar por el inicio de la Primera Guerra Mundial, fue un precedente fundamental para proyectos posteriores, que tuvieron su culminación con la celebración de la Exposición Internacional de 1929, que marcó un hito industrial y estético para la ciudad.

La Exposición de 1929 aportó un elemento fundamental: la luz eléctrica entendida como espectáculo y metáfora del progreso técnico, que alcanzó en ella un clímax de teatralidad y de prodigio inéditos hasta entonces $^{3}$. Integrada de forma dinámica y casi omnipresente en las arquitecturas del momento, la luz eléctrica se había convertido ya en el mayor símbolo de futurismo de la época. Las industrias modernas permitían e inspiraban la creación de nuevas formas y motivos decorativos, que transitaban entre el art déco y el racionalismo. En este sentido, la luz empezaba también a ser utilizada como elemento de reclamo publicitario y a ser introducida de manera ostentosa en aquellos edificios y establecimientos cuyas funciones se asociaban a la modernidad, como cines, comercios y salas de fiesta.

Grande fue también el impacto de la electrificación en las nuevas estructuras comerciales. Por un lado, el uso de iluminación eléctrica en los establecimientos comerciales generaba una imagen de modernidad y mejoraba el servicio de venta. Por el otro, favorecía la iluminación de espacios comerciales más amplios y complejos, como las galerías comerciales, que se extendieron por Europa durante las últimas décadas

${ }^{3}$ Pérez Rojas, J.: Art Deco en España, Madrid, 1990, Cuadernos Arte Cátedra, Capítu-
lo IX. 
del siglo XIX. Éstas ofrecían un nuevo modelo espacial y específico de consumo, en el que se concentraban pequeños comercios y servicios de ofertas variadas, a lo largo de paseos cubiertos que recorrían las plantas bajas de las parcelas edificadas, principalmente en los centros históricos de la ciudad ${ }^{4}$. Desde su origen, las galerías comerciales fabrican y simulan la estética y los usos de una auténtica calle comercial. En Barcelona, sin embargo, las galerías comerciales tardaron en implantarse y se difundieron en la década de los 40 , precisamente una etapa de máximo estancamiento económico y autarquía ${ }^{5}$. La iniciativa de la Avenida de la Luz en 1940 fue una auténtica precursora en la ciudad, que siguieron poco después las Galerías Manila también en 1940, ocupando el antiguo solar de los almacenes El Siglo, y dos años más tarde las galerías Maldá, o las galerías Condal ya en 1950. A pesar de estas precoces iniciativas, no fue hasta la década de los 80 cuando las galerías comerciales se desarrollaron ampliamente con casos como los del Bulevard Rosa o la Avenida, entre otras.

\section{La avenida de la Luz, una galería comercial moderna}

La Avenida de la Luz se encuentra junto a la Plaza de Cataluña, espacio que, tras varias tentativas y proyectos, fue finalmente diseñado y realizado por el arquitecto catalán Nebot en 1927. La plaza constituía ya por entonces un nudo de enlace entre las principales vías de comunicación del Ensanche y de la ciudad vieja, así como un privilegiado emplazamiento de monumentales edificios de banca y oficinas. A partir de su definitiva urbanización, la Plaza de Cataluña afianza su status como centro morfológico y funcional de la ciudad; como espacio articulador de movimientos urbanos, y como atractivo lugar de encuentro. Del mismo modo, se había ido configurando progresivamente como un nuevo espacio urbano de tráfico conflictivo, peligroso y de difícil resolución, tanto en superficie como en su subsuelo. Es a finales de los años 20 también, cuando se iniciaron las obras de interconexión entre las

${ }^{4}$ Carreras, C. (et al.): Barcelona, ciztat de compres. Les árees de concentració comercial a la ciutat de Barcelona, Barcelona 1990, Cambra Oficial de Comerç, Indústria i Navegació de Barcelona.

${ }_{5}^{5}$ Fabre, J. y Huertas, J. M.: Noticiari de Barcelona, Barcelona, 1991, Editorial La Campana. 
diversas estaciones de ferrocarriles subterráneos, afectando algunas de ellas a la Plaza de Cataluña y sus aledaños. En la década de 1930, al amparo de las complejas estrategias empresariales seguidas por las grandes industrias eléctricas que las sustentaban, se habían afianzado ya tres grandes compañías de transporte urbano, que habían hecho de la Plaza de Cataluña un importante nodo de interconexión de la red. Existían en ella cinco estaciones subterráneas, dispuestas en tres niveles distintos y sin relación entre sí, a las que llegaban y de las que partían 3 líneas urbanas y 2 de largo recorrido o suburbanas ${ }^{6}$.

La Avenida de la Luz, bajo la calle Pelayo y con entrada también por la Plaza de Cataluña, se situó en una de las múltiples galerías subterráneas de empresas de transporte ferroviario, que se habían construido a finales de los años 20 con el objetivo de facilitar los accesos y circulación peatonal a sus usuarios. La inarticulación entre los diferentes túneles y pasos de la arquitectura subterránea de la Plaza de Cataluña, y espacios contiguos como la Plaza Universidad y Urquinaona, estaban en la base del proyecto de la Ciudad de la Luz.

Denominar Avenida de la Luz a una galería de paso subterránea, iluminada por 216 tubos de neón de 40 watios, y revestida de una estética entre mágica y futurista que recogía la estela de luz dejada por la Gran Exposición de 1929 nos parece ahora, cuando menos, ambicioso (Fig. 1). No podemos pasar por alto el contexto económico y político en el que aparece, y el paisaje desolador de una ciudad destruida por las batallas. La Guerra Civil había irrumpido drásticamente en el desarrollo político, social y económico de todo el país. La entrada de las tropas franquistas en Barcelona el 26 de enero de 1939 significó el fin del conflicto bélico, pero las heridas de guerra eran tan visibles en un paisaje urbano desolado por las bombas y repleto de escombros, como en la erosionada y maltrecha vida social y económica de la ciudad. En los primeros años de dictadura se inició una etapa de reconstrucción asumida por el Ayuntamiento de postguerra y auspiciada por el régimen. La militarización de algunas empresas, la prestación de servicios urbanos mínimos y la reconstrucción de ciertas instalaciones y edificios, pretendían generar y alentar una imagen de aparente normalidad, e incluso de pres-

${ }^{6}$ Huertas, J. M. (et al.): La ciutat transportada, dos segles de transport col-lectiu al servei de Barcelona, Barcelona, 1997, Transports Metropolitans de Barcelona. Véase también: Miralles, C.: Transport i Ciutat, reflexió sobre la Barcelona contemporània, Bellaterra, 1997, UAB, p. 326. 
tigio para la ciudad, que quería ser transmitida por la Dictadura franquista y el Consistorio Municipal. Es en este escenario en el que se inaugura la Avenida de la Luz, al tiempo que se realizan obras de cierta envergadura y gran publicidad como la reforma el Paseo de Colón, o la recuperación de parte de la muralla romana.

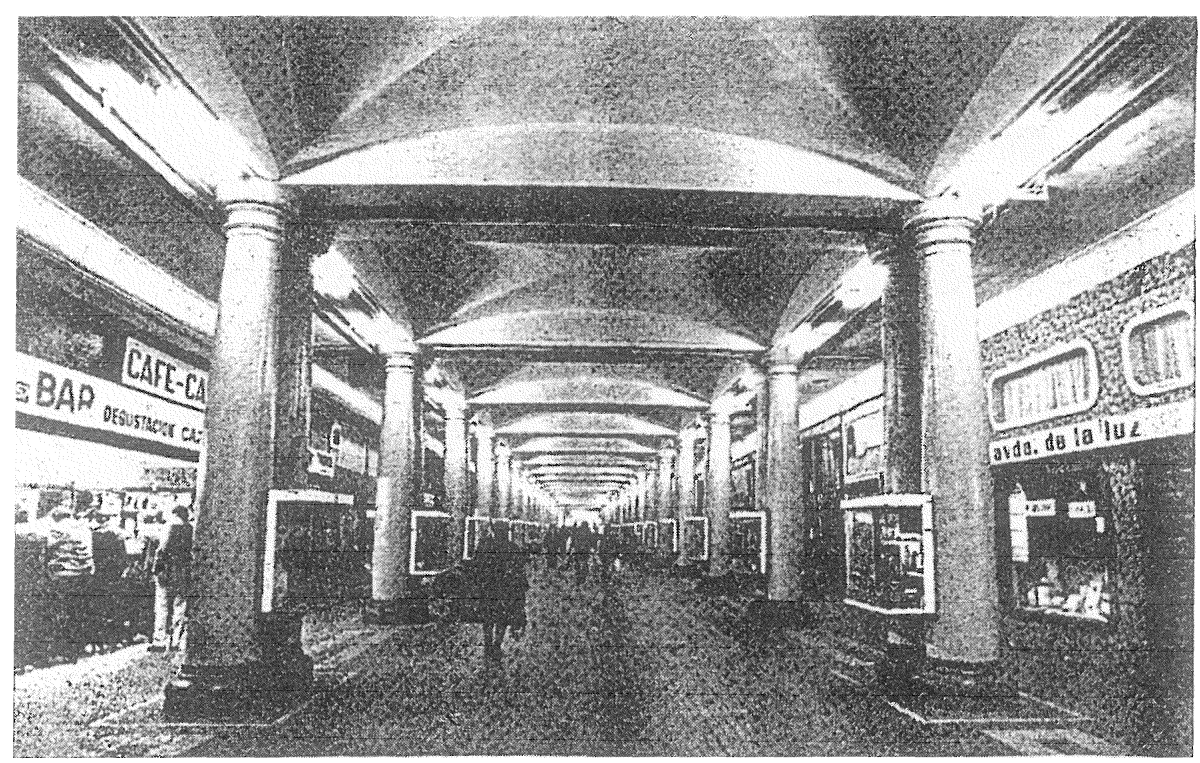

Fig. 1-Avenida de la Luz a finales de los 70 (foto Archivo de La Vanguardia).

Ambición era sin duda la característica que destilaba el proyecto de la Avenida de la Luz y la de su progenitor, Jaume Sabaté Quixal, un hombre de negocios y un visionario, de activa participación en la vida social de Barcelona, quien, entre otras actividades, sería luego fundador de la Cofradía del Arroz en 1969 y cónsul de Thailandia desde 1956. Para Sabaté, la Avenida de la Luz era sólo un anticipo de lo que él denominó la Ciudad Subterránea de la Luz, obra que nunca llegó a realizarse, aunque parte de ella se aprobó y fue publicada en el Boletín Oficial del Estado en fecha 15 de junio de $1953^{7}$.

${ }^{7}$ Boletín Oficial del Estado, Decreto de 5 de junio de 1953, por el que se declara de urgente construcción las obras autorizadas por el Excmo. Ayuntamiento de Barcelona a «Avenida de la Luz, S.A.», Madrid, 15 de junio de 1953, p. 3.647. 
La Avenida de la Luz ocupa el primero de los dos pisos subterráneos que conforman la estación de Plaza de Cataluña de los Ferrocarriles Catalanes, antigua compañía del Ferrocarril de Barcelona y Sarriá. Este piso, a siete metros por debajo de la calle Pelayo, es un espacio de 175 $\mathrm{m}$ de largo por 10 de ancho, con dos filas de grandes columnas pareadas de mármol que soportan el techo. Dispone de tres entradas, una por cada extremo de su plano, es decir, en la Plaza de Cataluña, en la confluencia de las calles Balmes y Pelayo, y en la calle Vergara. En los dos vestíbulos principales de estos accesos se encuentran las taquillas y las escaleras mecánicas que descienden al segundo piso de la estación. Éste se encuentra a $20 \mathrm{~m}$ bajo la superficie de la calle, y acoge las vías y los andenes que se reparten en tres naves, dos de ellas gemelas y paralelas, y una tercera ubicada bajo la calle Vergara.

Su ubicación gozaba de un carácter estratégico comercial indudable y oportuno. En primer lugar, era un lugar de paso para toda aquella población que se dirigía al tren de Sarriá o llegaba a la ciudad por este medio. Y en segundo lugar, abría una nueva vía, exclusiva para transeúntes, bajo la calle más comercial de Barcelona en los 40. Recordemos que en la calle Pelayo se encontraban, entre otros establecimientos, los primeros grandes almacenes de la ciudad desde la segunda década de los años veinte: El Siglo y El Águila. Y todo ello, además, en pleno centro de la urbe, junto a la Plaza de Cataluña, que une la ciudad vieja con el ensanche, como indicamos más arriba.

El atractivo de la Avenida radicaba sobre todo, en la aplicación de un modelo comercial poco difundido hasta el momento en la ciudad: la galería comercial. Una considerable y variada oferta de actividades se ponía a disposición del ciudadano que decidiese descender y atravesar aquella avenida de luz: desde el simple paseo y contemplación del espectáculo de modernidad, el consumo de artículos varios en algunas de las más prestigiosas firmas del momento, tomar un café, visitar su cine o exposiciones, dejarse caricaturizar por el dibujante Bon, o utilizar alguno de sus múltiples servicios. Un espacio tan semejante a una calle más de la ciudad, que pronto empezó a aparecer dibujado en las guías de Barcelona y todavía se reseña hoy como la calle subterránea de la ciudad, a pesar de su cierre primero y de su reciente reutilización como un espacio cerrado y tienda de la perfumería Sephora después.

La imagen generada por este espacio de consumo y la percepción que de él se tuvo, ha estado ligada a las diferentes etapas evolutivas por las 
que transcurrió. Durante la primera etapa de su existencia, cuando las crónicas de la época hablaban de lujo y solemnidad ${ }^{8}$, la Avenida contaba con 68 establecimientos entre los que había cafés, restaurantes, servicios postales y telegráficos, una peluquería, pastelerías, ultramarinos, joyerías, tiendas de ropa, perfumerías, etc., además de acreditadas firmas de la época como: Máquinas de Coser Sigma, Relojería Suiza, Patatas Miss, entre otras ${ }^{9}$. Era frecuente que la clase pudiente del momento se dirigiera a la Avenida para comprar electrodomésticos.

Las ofertas de ocio eran variadas. En uno de los locales próximos al vestíbulo de entrada por la calle Balmes, se abrió un estudio de cristal de Radio Nacional de España a la vista del público, que emitía noticias, música, programas y entrevistas realizadas por locutores que más tarde tuvieron gran éxito en la radio, como Enrique Casademont o Ricard Ardébol entre otros; también intervinieron artistas destacados como Lola Cabello, Enrique Borrás, Miguel Ligero o la arpista Rosa Balcells. Frente a este local, el 1 de enero de 1943 se inauguró uno de los primeros cines de la cadena Balañà que ofrecía incluso sesiones matinales. Por su parte, La Vanguardia había instalado una salita para reportajes. La Avenida de la Luz era un espacio vivo, muy frecuentado y con una imagen de modernidad, lo que alentaba iniciativas para la celebración de exposiciones (trenes en miniatura, soldaditos de plomo, etc.), o colocación de stands en el pasillo convirtiendo el lugar por unos días en una auténtica feria. Como punto de referencia en la ciudad, la Avenida de la Luz albergó en sus locales oficinas de información de algunos certámenes como el que tuvo lugar en la Feria Oficial e Internacional de Muestras sobre hostelería, gastronomía y alimentación celebrado en 1948.

Los visitantes diarios que transitaban por la Avenida de la Luz en su primera etapa se contabilizaban en unos 60.000 , según las crónicas periodísticas de la década de los 40 y 50 . Esta cifra hacia referencia tanto a los que cruzaban la Avenida para entrar en la estación de Plaza de Cataluña de Ferrocarriles a Sarriá, o los que acudían para «gozar del es-

8 «Acto inaugural de las obras de la Avenida de la Luz», La Vanguardia, 28 de julio de 1940; Sempronio: «El Apóstol del subsuelo», Diario de Barcelona, 31 de marzo de 1955. Véase también: AAAB: Expediente n. ${ }^{\circ}$ 134, Registre d'Expedients. Comissió Obres Públiques Eixample, Barcelona, 1940.

9 Archivo Administrativo del Ayuntamiento de Barcelona (AAAB): Sección Antecedentes, Exp. n. ${ }^{\circ}$ 62.199, Servicio de Edificación Particular, Barcelona, 1955. 
pectáculo con esa fantástica iluminación» y hacer uso de servicios generales «al abrigo de las inclemencias del tiempo» ${ }^{10}$. Los visitantes de la Avenida siguieron aumentando durante las primeras décadas de su existencia, a medida que los transportes subterráneos empezaban a destacar como medios más utilizados.

El éxito comercial y la singularidad urbanística de la Avenida de la Luz fue premiada por el Ayuntamiento de Barcelona, que declaró el lugar de atracción de forasteros y de turismo en virtud de un decreto de la alcaldía de fecha 23 de abril de 1949. También se hacen eco, además de las crónicas periodísticas de la ciudad, otras en el extranjero, como el periodista Eduard Miller, del New York Herald Times, quien en 1954 elogia la Avenida por su modernidad e innovación, donde «incluso hay un cine impecablemente limpio». Según Miller, «supera todo este concepto de vida al abrigo de la intemperie, porque se trata de una auténtica y amplia avenida ciudadana de nueva construcción» ${ }^{11}$. La influencia de la Avenida de la Luz se proyecto en otras ciudades europeas que quisieron seguir su ejemplo. Éste es al parecer, el caso de París, donde se habilitó una parada de metro de esta ciudad, concretamente la Ópera 3, para la instalación de comercios y servicios.

La estructura comercial, oferta, prestigio e imagen de la Avenida, sufrió un lento proceso de degradación y desatención a lo largo de sus cincuenta años de vida, que la condujeron a un inevitable deterioro. Así, en ese imaginario urbano compuesto de realidades objetivas y percepciones ciudadanas, a menudo borrosas, se han dibujado diversas y muy distintas descripciones de aquel espacio, lo que para unos fuera lujo, para otros decadencia y miseria, y para los que la vieron nacer y morir al cabo: la lenta crónica de una muerte anunciada.

\section{Las etapas de desarrollo: cincuenta años de vida de una calle}

La estructura arquitectónica que alberga la Avenida de la Luz que, como hemos dicho, forma parte del edificio de la estación de Plaza de Cataluña, tiene un origen algo confuso. Las referencias bibliográficas y

10 «La avenida de la luz, anticipo y promesa de la ciudad de la luz», Diario de Barcelona, 31 de diciembre de 1954. Y Carcasona, J.: «Una gran ciudad debajo de otra. Ocurrirá en Barcelona dentro de cinco años», La Vanguardia, 17 de abril de 1957.

11 Diario de Barcelona, op. cit., 1954. 
documentales en relación a la motivación y fecha de realización de este espacio son a menudo contradictorias y poco definidas. En esta circunstancia ha influido, sin dudas, el gran volumen y diversidad de obras subterráneas realizadas en las décadas de los 20 y 30 del presente siglo en Barcelona, que constituyeron una etapa de gran actividad financiera y constructiva en el sector de los transportes públicos subterráneos.

Así, el 26 de diciembre de 1924 se autorizaron los trabajos de soterramiento de la línea del Tren de Sarriá, que se inició finalmente el 1926. En 1929 el Tren de Barcelona a Sarriá se convirtió en el tercer metro de la ciudad, con una línea subterránea entre la Plaza de Cataluña y Sant Gervasi, inaugurándose la obra el 24 de marzo de dicho año. También en 1929 se inició un túnel de enlace (como igualmente hicieran otras compañías) entre el Metro Transversal y el Tren de Sarriá bajo la calle Pelayo. En 1932, el túnel se amplió con los pasillos de enlace entre el metro Transversal y el Gran Metropolitano ${ }^{12}$.

El espacio que ocupó la Avenida de la Luz tuvo su origen en la primera etapa de las obras realizadas para la conversión en subterráneo del Ferrocarril de Sarriá, concretamente entre 1926 y 1929; este último fue el año en el que apresuradamente se inauguró la estación o, mejor dicho, el segundo piso de ésta, quedando el primero, el que albergaría en el futuro la galería comercial, todavía en obras. La década de los años 30 fueron de transición para la Compañía del Ferrocarril de Sarriá, quedando paralizadas las actuaciones ${ }^{13}$.

A partir de 1936, durante el período bélico y bajo el control republicano, un comité obrero se hizo cargo de las compañías de transporte urbano, gestionando las redes y aportando algunas mejoras. En estos años, se inician unas obras de excavación sobre la calle Pelayo, con el objeto de enlazar los túneles de la estación del Metro Transversal en la Plaza de la Universidad con el Ferrocarril de Sarriá en la Plaza de Cataluña. Se perseguía la creación de una vía subterránea por la que movilizar de manera rápida y segura el material armamentístico y explosivo,

12 Huertas, J. M., (et al.), op. cit., 1997.

13 Véase Salmerón, C.: El tren de Sarriá. Historia del Ferrocarril Barcelona-Sarriá, In Els Trens de Catalunya, Barcelona, 1988, Ferrocarriles de la Generalitat de Catalunya, vol. XVIII. Préstese especial atención a la fotografía de archivo del autor, en la parte inferior de la página 32. Se trata del espacio que años más tarde ocuparía la Avenida de la Luz y que, en el momento de la fotografía, aparece como espacio de trabajo para las obras de construcción de la estación subterránea de la Plaza de Cataluña. 
desde el centro de la ciudad hasta los túneles de la Avenida Tibidabo, en la parte alta de la calle Balmes que ya estaban construidos aunque carecían aún de vías. Estos túneles se utilizaron posteriormente como almacén de material de guerra. Al término de ésta, las obras se encontraban en avanzado estado de ejecución, quedando una enorme brecha abierta sobre la calle Pelayo, que fue aprovechada para la creación de una segunda entrada en el Metro Transversal, sito bajo la plaza Universidad $^{14}$.

En esta etapa, el Ayuntamiento promovió el reinicio de las obras para la completa transformación en subterráneo de las líneas. En el caso de la compañía de Ferrocarril de Sarriá, las diversas modificaciones del proyecto inicial y su adecuación a las perspectivas urbanísticas del momento hicieron que se retardara su inicio todavía unos años, hasta que el 29 de noviembre de 1946 se empezaron de nuevo las obras ${ }^{15}$.

De entre los diversos proyectos que habían quedado pendientes para esta empresa ferroviaria, se contemplaba también la construcción de una amplia sala-vestíbulo subterráneo, que permitiese unir los diversos accesos de la estación de la Plaza de Cataluña de dicha Compañía. En el año 1940, la obra se encontraba en avanzado estado de ejecución, y sólo requería instalaciones de alumbrado y decoración ${ }^{16}$, que no habían podido realizarse por haber sido utilizada la sala como espacio de trabajo para la terminación de la estación.

Ese mismo año, se firmó un convenio entre la Compañía de Ferrocarril y un grupo industrial de Barcelona denominado S.A.T., S.L. sin intervención alguna del Ayuntamiento, para la ejecución en aquella sala de obras e instalaciones que permitiesen el acondicionamiento del espacio. La sociedad S.A.T. se constituye el 3 de mayo de 1940 con socios y capital local ${ }^{17}$. Diez días después, el 13 de mayo, se inicia la relación arrendaticia entre ambos grupo por el alquiler de parte del vestíbulo de la estación de la Plaza de Cataluña, delimitado por las entradas o ac-

${ }_{14}$ Archivo del Colegio de Arquitectos de Cataluña: «Obra realizada por el primer Ayuntamiento de Barcelona después de la liberación», Memoria 1939-44, Comisión Especial del Ensanche Barcelona, Barcelona, 1944.

${ }^{15}$ Salmerón, C.: Op. cit., 1988. 1956.

${ }^{16}$ AAAB: Exp. n. ${ }^{\circ}$ 658, Comisión Obras Públicas, Negociado Obras Públicas, Barcelona,

${ }_{17}$ Registro Mercantil de Barcelona: Avenida de la Luz, S.L., hoja n. ${ }^{\circ}$ 20.091. Los socios fundadores fueron: Jaime Sabaté (comercial), Andreu Baiget (pintor), Juan Toldrá (industrial). 
cesos sitos en las calles Vergara, Pelayo, y la misma plaza. El contrato ${ }^{18}$ establecía una duración indefinida del mismo y la capacidad explícita de la sociedad para subarrendar el local.

El 27 de julio de 1940 se realizó el acto inaugural de las obras de adecuación de lo que habría de ser la futura Avenida de la Luz. El proyecto incluía entonces la creación de 60 establecimientos comerciales, un salón de cine, fiestas y exposiciones, así como diversos bares. Las obras se realizaron con gran agilidad, y en cuatro meses el espacio estaba perfectamente acondicionado. El coste total fue de 30 millones de pesetas, que incluían la iluminación y decoración del espacio.

El 29 de octubre del mismo año, un día antes de la inauguración del local y con motivo de la próxima asistencia al acto del alcalde Miguel Mateu, se otorgaba una autorización municipal, y se iniciaba un proceso para legalizar la relación de arriendo y la propia existencia de la Avenida de la Luz. La autorización municipal destacaba el carácter provisional de lo actuado hasta que la Compañía Ferroviaria y el Ayuntamiento llegaran a una solución mixta, es decir, un arreglo contractual en el que ambas partes salieran beneficiadas ${ }^{19}$. Las relaciones entre estas dos entidades van a marcar el desarrollo y evolución de la Avenida de la Luz, desde el mismo día en que se abrió hasta su cierre.

Finalmente, el 30 de octubre de 1940, se realizó el acto inaugural de la Avenida de la Luz, con la asistencia de todas las autoridades, militares, políticas y eclesiásticas locales, así como con la presencia del general Mújica en representación del gobierno dictatorial. Asistió además al acto Vidal Burnils, del departamento comercial de la Compañía Barcelonesa de Electricidad, lo que es probablemente una muestra del interés que esta empresa tenía en el proyecto comercial.

En total habían transcurrido seis meses de obras y relaciones ágiles para la constitución de la que habría de ser una de las primeras galerías comerciales de Barcelona. Un corto período, si se tiene en cuenta el decadente escenario económico y social que dominaba la ciudad de postguerra.

Las décadas de 1940 y 50 constituyeron el mejor período para la salud comercial y atractivo de la Avenida. El 25 de junio de 1943 la so-

\footnotetext{
18 Archivo privado Vilardebó, A.: Contrato legal de arrendamiento de locales. Y Demanda interpuesta por el gabinete Vergés-Mas en representación de la Asociación de Comerciantes Avenida de la Luz.

${ }_{19}$ AAAB, op. cit., 1956. Exp. 658.
} 
ciedad gestora S.A.T., S.L. cambia su nombre por el de Avenida de la Luz, S.L. Eran años de prestigio, modernidad y gran actividad de la galería. Años en los que el proyecto más ambicioso de la Ciudad Subterránea de la Luz parecía inminente como reflejaban los artículos periodísticos de la época y las declaraciones que aparecían. Se escriben titulares como: Nuestra futura Gran Ciudad de la Luz, La Avenida de la Luz, anticipo y promesa de la ciudad de la luz, Una gran ciudad debajo de otra. Ocurrirá en Barcelona dentro de cinco años, La Avenida de la Luz, centro turistico de primer orden, etc. ${ }^{20}$.

En este período, concretamente el 3 de junio de 1955, la empresa gestora adapta sus estatutos a la vigente Ley de Sociedades de Responsabilidad Limitada. El mismo mes, Jaime Sabaté Quixal, auténtico promotor del proyecto empresarial que analizamos, es nombrado gerente y administrador de Avenida de la Luz, S.L. ${ }^{21}$. Poco tiempo después, los principales accionistas de la sociedad pasan a ser Sabaté y los dos hijos de éste.

Paralelamente, el 11 de julio de 1955 acababa el plazo de noventa y nueve años que tenía la Compañía Ferroviaria para explotar el Tren de Sarriá, y la propiedad de sus instalaciones revertía al Ayuntamiento de Barcelona según el Real Decreto de 26 de diciembre de 1924. Y, aunque se prolongó el período de reversión por treinta años más, el hecho tuvo una influencia clave en la Avenida de la Luz, que pasó a ser considerada una calle más de la ciudad. Según un acto celebrado el 7 de diciembre de 1956 en la misma Avenida, el espacio pasaba a incorporarse plenamente a la jurisdicción municipal ${ }^{22}$. A la celebración asistió el alcalde señor Molleví quién, entre otras cosas, expresó el apoyo municipal a la futura Ciudad Subterránea de la Luz, mientras una maqueta iluminada de éstas se exponía ante el público. Lo cierto es que, la por entonces famosa y prestigiosa galería comercial, aparecía señalada en todas las guías municipales de la ciudad.

${ }^{20}$ Lorca, A.: «Nuestra futura «Gran Ciudad de la Luz»», Gaceta Municipal, n. ${ }^{\circ}$ 5, Ayuntamiento de Barcelona, Barcelona, febrero de 1954; Vázquez Prada, F.: «La Avenida de la Luz, centro turístico de primer orden», La Prensa, 23 de julio de 1957; Diario de Barcelona, op. cit., 31 de diciembre de 1954; Carcasona, J.: Op. cit., 1957.

${ }^{21}$ Registro Mercantil de Barcelona: Avenida de la Luz, S.L.

${ }_{22}$ Vázquez Prada, F.: «Barcelona ha ganado una calle», La Prensa, 8 de diciembre de 1956; Vázquez Prada, F.: «La Avenida de la Luz, incorporada plenamente a la jurisdicción municipal», La Vanguardia, 8 de diciembre de 1956. Véase también Vidal, D.: «La Avenida de la Luz va a cumplir veinticinco años», TeleXpress, 19 de febrero de 1965.

$$
-500-
$$


Esa nueva condición de espacio público, y consideración de calle de la ciudad al lugar que ocupaba la galería comercial, tenía cierta ambigüedad e indefinición por las implicaciones y responsabilidades reales que ello suponía, especialmente si tenemos en cuenta que Avenida de la Luz, S.L. seguía manteniendo una relación contractual con la Compañía Ferroviaria y pagando un alquiler por el espacio ocupado. Esta característica se mantuvo hasta el cierre del pasaje, mientras que el mantenimiento, limpieza y seguridad de la nueva calle era una responsabilidad que ninguna de las entidades asumía con claridad.

La década de los 60 fue una etapa de transición en la que la indefinición del espacio y ambigüedad en las responsabilidades y gestión se acentuaron. Empezaba un proceso de lenta sustitución de comercios. La que antes fuera una dinámica emisora de radio abierta al público, en esta etapa es $L a$ Voz, una estación de altavoces que difunde únicamente música y anuncios. La dirección decide variar la orientación de la galería comercial hacia un público de clases medias y medias-bajas. En este período se contabiliza una media de 40.000 visitantes diarios. Lentamente, deja de ser noticia y motivo de comentarios público el proyecto de la Ciudad Subterránea.

A partir de los años 70, la Avenida entra en su última etapa, y es testigo de un lento proceso de abandono por parte de todas las instituciones implicadas, al tiempo que empeoraban las condiciones de higiene y seguridad. Existían conflictos para decidir la responsabilidad del mantenimiento del paso subterráneo, que se convertía paulatinamente en refugio de indigentes. Con todo, el 1 de enero de 1972 la relación arrendaticia fue objeto de renovación mediante el correspondiente contrato que sustituía al anterior. El alquiler ascendía a 520.000 pesetas anuales, y era revisado cada cinco años. En los últimos años y hasta su reciente extinción, la relación arrendaticia se mantenía con la empresa pública Ferrocarriles de la Generalitat de Cataluña (FGC), que desde la publicación del Decreto de la Generalitat de 7 de diciembre de 1979, se hizo cargo de las líneas antes explotadas por Ferrocarriles de Vía Estrecha, S.A. (FEVE), y de Ferrocarril de Barcelona a Sarriá, S. A. ${ }^{23}$.

$\mathrm{El}$ deterioro y abandono que sufría la galería generaba una imagen negativa y la percepción de espacio peligroso, que procuraba ser evitado

${ }^{23}$ Salmerón, C.: Op. cit., 1988. 
por los peatones que se dirigían a la estación ferroviaria. Muchos de los establecimientos comerciales empezaron a anunciar su traspaso. En 1988 quedaban tan solo 38 locales deteriorados, de los cuales 27 se mantenían en funcionamiento, siendo los más visitados los bares, unos billares y el cine que, desde marzo de 1988 se dedicó a proyectar películas pornográficas. Los artículos periodísticos describen un panorama desolador de la situación comercial que vivía la galería. Titulares como: La Avenida de la Luz se muere, La Avenida de la Miseria ${ }^{24}$, relatan una situación de crisis y nostalgia de la que «debía ser una joya de Barcelona». El mismo Sabaté con 86 años de edad declaró en una entrevista realizada en 1987 su desilusión por el proyecto y la asunción de su parálisis: «Yo siempre he sido un somiatruites (...)», «y ya ve, la cosa quedó en un pasillo. Un pasillo que no va a ninguna parte» ${ }^{25}$.

En este tiempo surgieron algunos proyectos de rehabilitación pero ninguno llegaría a prosperar. Especialmente destacado es el que presentó el Gremio de Artesanos en 1984, por el cual se pretendía la creación de una Avenida de la Artesanía, reconvirtiendo las tiendas en talleres. En 1986 Jaime Sabaté Quixal, ya anciano, abandona la sociedad que lideraba, y la gestión pasa a manos de Dolores García Calviño, con Jorge Vidal Cairat como apoderado, que la mantendrán durante no más de dos años antes de vender la sociedad a un nuevo comprador.

Durante los últimos años, a finales de los 80, ante el evidente abandono que sufre la galería, la Asociación de Comerciantes de la Avenida empezó a manifestar su malestar y a exigir responsabilidades a las entidades implicadas. En esta etapa existió una gran indefinición por parte del Ayuntamiento respecto a las actuaciones que debían tomarse, aunque se asumía que la Avenida debía ser remodelada dentro de un amplio proyecto que abarcara toda la manzana de la calle Pelayo. El céntrico so-

${ }^{24}$ Bru Rovira: «La Avenida de la Miseria», La Vanguardia, 22 de marzo de 1987; González Cabezas: «La Avenida de la Luz se muere», El Periódico, 4 de mayo de 1979. Véase también los artículos: «L'Avinguda de la Llum, 48 anys de degradació. La galeria comercial passa una greu crisi», Diari de Barcelona, 20 de marzo de 1988; «La Avenida de la Luz a oscuras», El Periódico, 30 de diciembre de 1991. «Avenida sin luz y sin cine», La Vanguardia, 4 de enero de 1993.

${ }_{25}$ Bru Rovira: Op cit., 22 de marzo de 1987. Véase también: «La ordenación de la calle de Pelayo debe ser una solución de conjunto», El Correo Catalán, 21 de marzo de 1954. AAAB: Exp. n. ${ }^{\circ}$ 679, Registre d'Expedients, Comissió Obres Públiques Eixample, Barcelona, 1928. 
lar, también denominado popularmente como «triángulo de la vergüenza» por el fracaso de todos los proyectos urbanísticos que trataron realizarse en él, permanecía todavía sin edificar y ocupado parcialmente por un aparcamiento de la Guardia Urbana.

Finalmente, el 2 de febrero de 1988 el Ayuntamiento y los Ferrocarriles de la Generalitat de Catalunya, presentan un proyecto conjunto para la construcción de un complejo hotelero y comercial en el solar de la calle Pelayo, lo que comportaba la desaparición de la Avenida de la Luz y su transformación en un aparcamiento, una idea que fue variando en los últimos años. La empresa Estudis d'Espais Urbans, firma de capital riesgo en la que participan destacados comerciantes de la ciudad como Enric Vives, así como la empresa municipal Iniciatives, S.A., ganó el concurso para llevar a cabo esa remodelación. El Ayuntamiento impuso como condición para edificar el solar, que la empresa contemplara el futuro de la Avenida, y una solución para los arrendatarios de los locales.

Quince días después, el Ayuntamiento requirió a FCG que acabara definitivamente con la situación ilegal que representa el arriendo a Avenida de la Luz, S.L. de los espacios que constituyen las galerías comerciales, y pusieran fin a los subarriendos y ocupaciones particulares que se derivaban. Se instaba a que aquel espacio fuera devuelto al Ayuntamiento. Así, el 4 de marzo del mismo año, los FGC reclaman por escrito a AL, S.L. que les devuelvan los espacios ocupados vacíos y aluden a la invalidez del contrato otorgado el 1 de enero de 1972.

En esa coyuntura de presión del consistorio municipal, y de la empresa ferroviaria, así como de deterioro de la galería, la Avenida de la Luz vuelve a cambiar de manos, y el 1 de diciembre de 1988, Enrique Vives Valls, quien como hemos indicado también pertenecía a la sociedad Estudis d'Espais Urbans y, por lo tanto, parte interesada en el proyecto inmobiliario, pasó a ser el administrador de la sociedad AL, S.L., pudiendo conferir toda suerte de poderes judiciales y extrajudiciales y revocarlos, incluyendo el de instar desahucios.

Ante la compleja situación provocada y las protestas de los comerciantes, se estableció un protocolo entre el Ayuntamiento y FCG, por el cual se indemnizaba a los comerciantes que estaban ubicados bajo la manzana por edificar. Sin embargo, quedaron una decena de tiendas en el vestíbulo de la estación del tren. Estas últimas recibieron una orden de desahucio, ante la cual presentaron una demanda contra todas las en- 
tidades implicadas, a fin de ser reconocidos como los auténticos arrendatarios de los espacios que ocupaban, en lugar de Avenida de la Luz, S.L. Sin embargo, la sentencia judicial ${ }^{26}$ desestimó la demanda interpuestas, y los últimos comerciantes fueron desahuciados con una escasa indemnización.

En 1990, a los cincuenta años de su apertura, se cerró la única calle subterránea de Barcelona y una de las primeras galerías comerciales de la ciudad. Tras tapiar la Avenida de la Luz, el camino quedaba expedito para la construcción el complejo comercial que habría de ocupar el solar que ya entonces empezaba a denominarse el triángulo de oro. Este proyecto, empezó a tomar forma a finales de la década de los 80, por iniciativa de capital francés, concretamente de las inmobiliarias VandaelePierre, quienes iniciaron una labor de compra sistemática de las fincas del solar. En 1992 adquirieron la concesión de uso comercial para el lugar, que habían obtenido poco tiempo antes el grupo de comerciantes de la ciudad al que nos referíamos más arriba, encabezados por Enrique Vives. El proyecto consiguió superar algunos problemas de tipo económico, y una moratoria comercial interpuesta por la Generalitat que bloquearon la iniciativa. La aseguradora UAP primero, y la fusión de éstas con AXA después, asumieron la promoción del complejo comercial El Triangle. El proyecto, a cargo de los arquitectos Cristian Cirici y Carles Bassó, fue inaugurado el 12 de noviembre de $1998^{27}$, y tuvo un coste total de 20.000 millones de pesetas. AXA es uno de los grupos aseguradores más importantes de Europa que, de la misma forma que Winterthur con el shoping center de L'Illa Diagonal, ha querido invertir en el sector comercial de Barcelona.

El espacio que ocupaba la Avenida de la Luz, ha vuelto a ser parcialmente reutilizado como espacio de consumo. La firma francesa Sephora ha instalado allí la tienda más importante de productos cos-

26 Sentencia, del Juzgado de Primera Instancia n. ${ }^{\circ} 27$, Barcelona, ref. 127-90, 22 de abril de 1991. Véanse también los artículos: «Una batalla legal oscurece la Avenida de la Luz», La Vanguardia, 7 de julio de 1988; Tarín, Santiago: «El último que apague la luz, recta final de las negociaciones para cerrar los comercios de la Avenida de la Luz», La Vanguardia, 21 de mayo de 1990.

27 Ricart, M.: «El Triangle alza el telón», La Vanguardia, 11 de noviembre de 1988. Véase también: «Primera piedra del complejo lúdico del «triángulo de oro», en El Periódico, 19 de abril de 1997; y Huertas, J. M.: «De la Luz al Perfume», en El Periódico, 6 de diciembre de 1988. Como antecedente de la urbanización del solar existente sobre la Avenida de la Luz véase: AAAB: Exp. n. ${ }^{\circ}$ 2.036, Comisión Obras Públicas, Negociado Obras Públicas, Barcelona, 1960. 
méticos en Barcelona, tanto por su extensión como por la variedad de la oferta. Sin embargo, a pesar de la operación de maquillaje que ha sufrido la Avenida de la Luz, el bloqueo de una de sus entradas, ha hecho que se rompiera la continuidad del espacio, y su original carácter de calle subterránea de la ciudad.

\section{La Ciudad Subterránea de la Luz}

La Avenida de la Luz era sólo un anticipo de lo que debía ser la Ciudad Subterránea de la Luz, proyecto ideado e impulsado por Jaime Sabaté. Dicho proyecto, que nunca llegó a prosperar, estuvo siempre en el ánimo de su progenitor como lo demuestra el hecho de realizar hasta cinco peticiones formales al Ayuntamiento, acompañadas de planos, presupuestos y empresa constructora. Una maqueta de la Ciudad Subterránea estaba colocada en la Avenida y fue mostrada en diversos actos y celebraciones a autoridades locales y extranjeras. Durante las primeras etapas de vida de la galería comercial, su construcción parecía inminente, hasta que empezó a ser abandonado y considerado una locura. Sin embargo, no se trataba de un proyecto aislado, sino que estaba imbuido de las influencias del arte racionalista de los años 30, con proyectos colosales y utópicos, como la Nave Luminosa de Buigas entre otros.

Como hemos destacado anteriormente, en los años 40 la Plaza de Cataluña empezaba a ser un verdadero nudo de enlace entre las principales vías de comunicación del ensanche y de la ciudad vieja, configurándose progresivamente como un espacio de tráfico conflictivo, peligroso y de difícil resolución. Y no solamente en la superficie. La complejidad de unidades subterráneas bajo la plaza y las dificultades espaciales para imaginarlas ensambladas entre sí, están en la base y origen del proyecto de la Ciudad Subterránea de la Luz, que debía articular y dar sentido a todo ese espacio subterráneo. Además, el proyecto pretendía dotar a la plaza de una renovada oferta de «subsuelo» destinado a usos comerciales y de servicios ${ }^{28}$ (Fig. 2).

\footnotetext{
${ }^{28}$ Sabaté Quixal, J.: «¿Cómo será la Barcelona el año 2000?», Gaceta Municipal, n. ${ }^{\circ}$ 31, Ayuntamiento de Barcelona, Barcelona, julio de 1957. 


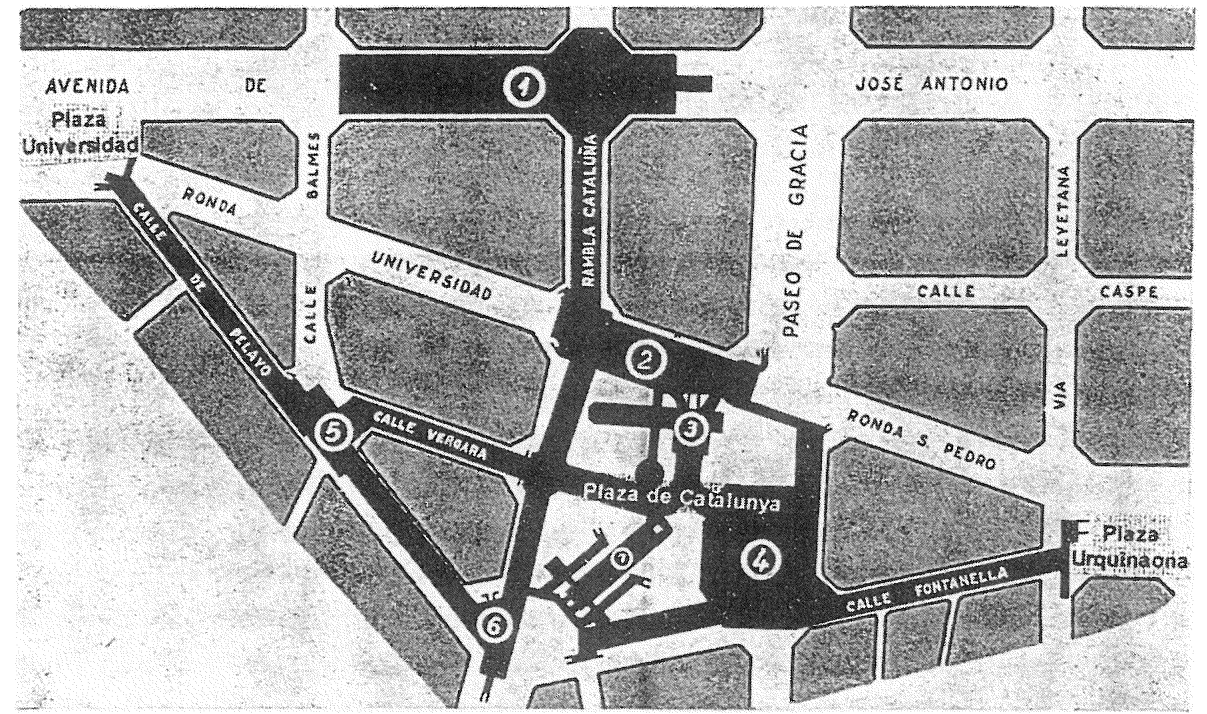

Fig. 2.-Plano de la Ciudad Subterránea de la Luz en el que se indican sus diversas instalaciones, 1954. 1) Estacionamiento. 2) Enlace con los vestíbulos de RENFE y Metro Transversal. 3) Enlace con vestíbulo de la Guardia Urbana, salón de exposiciones, y salida central. 4) Palacio de deportes y espectáculos. 5) Avenida de la Luz, sector comercial y enlace con la Avenida Tibidabo. 6) Enlace con vestíbulo del ferrocarril de Sarriá. 7) Enlace con el vestíbulo del Gran Metro (según A. Lorca).

Algunas de estas ideas aparecen ya en el origen y desarrollo de los transportes subterráneos en Barcelona, y constituyen un precedente teórico a algunos de los objetivos perseguidos por la Ciudad Subterránea de la Luz. Fue al iniciarse el siglo xx, cuando aparecieron las primeras iniciativas y proyectos concretos en relación al diseño de líneas de transporte ferroviario subterráneo en la ciudad. En 1912 se presentan y aprueban los dos primeros proyectos de construcción de líneas. En primer lugar, el ingeniero Fernando Reyes recibe la concesión de una línea subterránea, de orientación sudoeste a noreste que atravesaba la ciudad de manera transversal. Reyes buscaba unir las principales estaciones de tren de la ciudad (de Francia y del Norte, que pertenecían a Compañías distintas), a fin de facilitar lo que en términos actuales entendemos por nodos intermodales, es decir, permitir la correspondencia 
entre distintos modos de transporte, buscando una red coherente con las necesidades de movilidad ciudadana. Además, mediante una gran estación en la Plaza de Cataluña, se pretendía centralizar todo el tráfico ferroviario en ese punto de la ciudad, donde también encontraría un enlace rápido con el tren de Sarriá. También en 1912 se aprueba un proyecto y se otorga una concesión a los ingenieros Pau Müller y Gonçal Zaragoza, de una línea de ferrocarril subterránea que recorría la ciudad en orientación de mar a montaña. En ambos casos, la falta de capital riesgo para la financiación supuso la posposición de los proyectos.

La década de los 20 y 30 del presente siglo constituyeron una etapa de gran actividad financiero-asociativa y constructiva en el sector de los transportes públicos subterráneos. El 17 de diciembre de 1920 un grupo de empresarios catalanes, con participación financiera vasco-francesa, crearon la sociedad Ferrocarril Metropolitano de Barcelona, S.A. e hicieron suya la concesión y el proyecto de Fernando Reyes. Dos años después, «a tenor de la importancia del proyecto para la ciudad, el Ayuntamiento adquirió un paquete importante de acciones, que convierten la empresa en casi semipública» ${ }^{29}$. Por otro lado, el 26 de mayo de 1921 un grupo de empresas creó la sociedad Gran Metropolitano de Barcelona, S.A. (Gran Metro). Partiendo, aunque con algunas variaciones, del proyecto de los ingenieros Pau Müller y Gonçal Zaragoza, el 30 de diciembre de 1924 inauguran el tramo Lesseps-Cataluña. Paralelamente, el 26 de diciembre de 1924 se autorizaron los trabajos de soterramiento de la línea del Tren de Sarriá, iniciándose finalmente, en 1926. En 1929 el Tren de Barcelona a Sarriá se convirtió en el tercer metro de la ciudad, con su línea subterránea entre la Plaza de Cataluña y Sant Gervasi, inaugurándose la obra el 24 de marzo de dicho año.

La Ciudad Subterránea se planteaba así como una solución a este problema de la enorme complejidad de estructuras subterráneas, ofreciéndose como un espacio de interconexión e integración entre las diferentes redes de transporte, bajo el subsuelo del centro de la ciudad, uniendo las Plazas de Cataluña con las de la Universidad y Urquinaona. El proyecto pretendía dar respuesta a la necesidad de comunicar los tres nudos más importantes de circulación subterráneas, enlazando así los distintos transportes subterráneos: Gran Metro Transversal, Ferrocarril del Norte, Sarriá y Ferrocarriles Catalanes. Tan necesaria e inminente pa-

${ }^{29}$ Huertas, J. M., (et al.), op. cit., 1997. 
recía la construcción de esta obra, que en declaraciones realizadas por el alcalde de Barcelona en Madrid afirmaba: «uno de los objetivos principales consiste en coordinar túneles existentes comunicados entre sí, a fin que formaran una sola red, a la cual quedará incorporada la Avenida de la Luz, El Metro Transversal, el Gran Metro, y el Ferrocarril de Sarriá podrán realizar intercambio de viajeros» ${ }^{30}$.

Desde el punto de vista urbanístico, la Ciudad Subterránea ofrecía las ventajas de lo que por entonces era conocido como la doble calle, es decir zonificar verticalmente el espacio urbano. Estas «ingeniosa» solución a los crecientes problemas de la circulación urbana permitía así un paso subterráneo exclusivo para los peatones, libre de los ruidos, molestias y, sobre todo, al abrigo de las inclemencias del tiempo y lejos de los accidentes de tráfico tan frecuentes en ese período. Mientras tanto, la circulación rodada permanecía en superficie.

El proyecto de Sabaté pretendía aprovechar los vestíbulos subterráneos de las grandes Compañías Ferroviarias bajo las Plazas de Cataluña, Universidad y Urquinaona, y unirlos entre sí con la Avenida de la Luz, otorgando una continuidad y coherencia a todo el espacio del subsuelo. El objetivo de todo ello era centralizar los transportes a fin de ahorrar tiempo en los trasbordos y evitar los riesgos de circular en superficie. Recordemos que la ordenación del tráfico rodado en las décadas del 40 y 50 era todavía ineficaz, lo que motivaba múltiples accidentes. Además, se buscabas centralizar también la oferta de servicios públicos como Correos, Telégrafos y Radio, instalaciones que debían ocupar su lugar en la moderna ciudad soterrada. Los pasillos iban a estar dotados de una oferta comercial y de ocio en la línea de la Avenida. Se argüía como apertura de nuevos espacios y recuperación de las actividades comerciales en el centro de la ciudad, que en ese momento había sido desplazado a otras áreas en beneficio de las actividades bancarias. Además, «incorporar un salón para exposición permanente de las últimas novedades industriales y comerciales del mundo» ${ }^{31}$, así como también, y en este punto se aprecia la grandilocuencia del proyecto, un palacio de deportes con piscina para competición internacional. A fin de favorecer la accesibilidad a este espacio se proyectaba un aparcamiento bajo la que fuera la Avenida José Antonio, entre Balmes y Paseo de Gracia (Fig. 3).

${ }^{30}$ Del Castillo, J.: «Los enlaces subterráneos de las comunicaciones de la Plaza de Cataluña vuelven a la actualidad», La Solidaridad, 6 de abril de 1957.

31 Lorca, A.: Op. cit., 1954. 

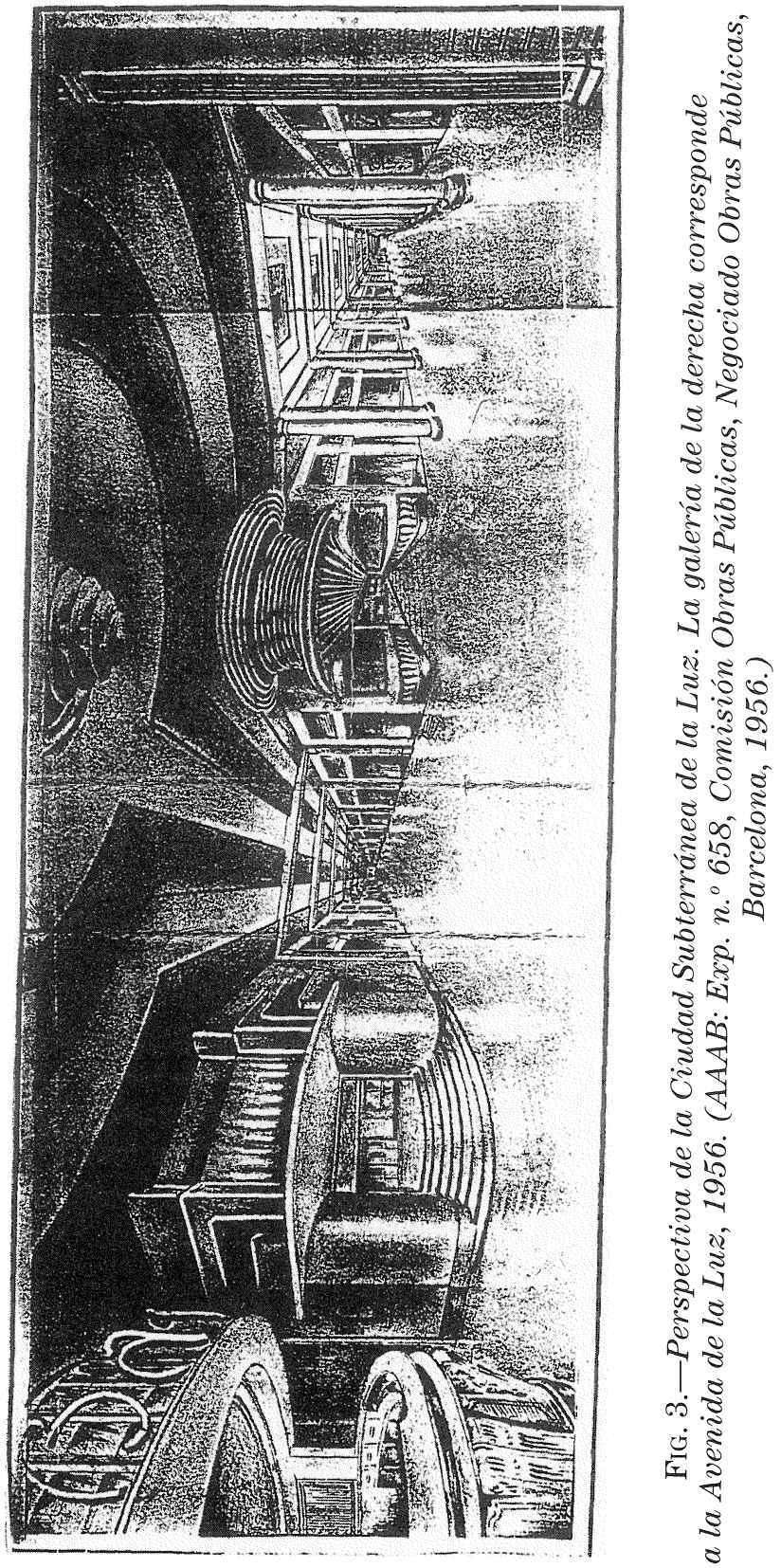

$-509-$ 
La superficie total que incluía el proyecto era de 14.000 metros cuadrados. Se preveía el acceso al primer piso subterráneo que constituiría la ciudad por 17 escaleras mecánicas distribuidas por todo el perímetro. Para la primera sección de la ciudad subterránea se calculó un coste de 30 millones de pesetas y un plazo de dos años para su ejecución. Para su conjunto se estimó un coste total de 200 millones de pesetas y diez años de terminio. El plan técnico había sido realizado por los ingenieros Conde, Folch y Ríos. El plan de ampliación fue aprobado por el Ayuntamiento y de conformidad con la Compañía Ferroviaria. La primera etapa de ampliación llegó a aparecer aprobada y con carácter de urgencia para su realización el Boletín Oficial del Estado con fecha 15 de junio de 1953, según un decreto del Ministerio de Obras Públicas.

Con todo, el proyecto nunca llegaría a realizarse, principalmente por complicaciones que hubo a nivel económico y por el vacío de competencias en el subsuelo, con una difícil atribución de responsabilidades en relación al pago y reparto de tareas.

\section{Conclusiones}

El artículo ha querido mostrar el origen y desarrollo de la Avenida de la Luz, atendiendo a la significación que las iniciativas privadas y públicas, así como la política urbana de cada momento histórico, han tenido en ella. De este modo, se ha puesto de manifiesto la clara incidencia de estos agentes en la configuración de la morfología y dinámica urbana.

La Avenida de la Luz, fue un exponente de modernidad y lujo en la Barcelona de los años 40 y 50, utilizada por el Ayuntamiento de postguerra como imagen de normalidad y progreso en tiempos de crisis y convulsión social. En un ambiente político favorable, Jaime Sabaté Quixal puso en marcha la instalación de una galería comercial en Barcelona, aportando un modelo de espacio de comercio y consumo poco conocido en la ciudad, y cuyo éxito inspiró iniciativas posteriores.

El uso de la electricidad, no sólo como mejorada capacidad técnica para la iluminación de espacios comerciales, sino también como nueva estética, símbolo de futurismo y calidad de vida, contribuyó en gran medida al efecto publicitario y de imagen moderna que se perseguía, expresada incluso en el propio nombre de la galería. La Compañía Barce- 
lonesa de Electricidad se mostró interesada por el proyecto. Hay que tener en cuenta que coincidiendo con los primeros años de la galería, las estrategias empresariales de las grandes Compañías de electricidad se orientaban hacia cuestiones referentes a la comercialización y a la captación de nuevos consumidores y que, en este sentido, la Avenida de la Luz constituyó un negocio atractivo tanto por el consumo que ella misma realizaba como por el que generaba.

La Avenida de la Luz fue innovadora también en el aprovechamiento y uso comercial de espacios subterráneos en Barcelona; espacios que habían sido creados a partir del soterramiento de los transportes ferroviarios. Desde el punto de vista urbanístico, la iniciativa de Sabaté aportó a la ciudad el concepto de «doble calle», dando respuesta a la que fuera una complicada y peligrosa circulación peatonal en superficie, mediante el acondicionamiento de una auténtica vía subterránea. Poco tiempo después de su inauguración. La Avenida de la Luz fue reconocida por el municipio como la calle subterráneas de Barcelona. Sin embargo, el ambicioso proyecto de la Ciudad Subterránea de la Luz que debía dar continuidad a la Avenida, no llegó nunca a prosperar debido a problemas técnicos, económicos, y al vacío legal existente respecto al subsuelo de la ciudad. La Avenida de la Luz ha sido, y en cierta manera todavía es, un espacio singular y destacado de Barcelona, tanto por su condición de calle subterránea, como por su peculiar evolución histórica y los diferentes usos que ha acogido en su interior. Desde noviembre de 1998 una multinacional ha vuelto a iluminar de modernidad las columnas de la que, para el barcelonés, ya siempre será la Avenida de la Luz.

\section{Fuentes Documentales}

Archivo Administrativo del Ayuntamiento de Barcelona (AAB)

Archivo Histórico del Ayuntamiento de Barcelona

Hemeroteca de La Vanguardia

Archivo privado de Jaime Sabaté Herce (hijo de Jaume Sabaté Quixal) Archivo privado de Emilia Jover Bardolet (segunda esposa de Jaume Sabaté Quixal)

Archivo Privado de Asumpta Vilardebó (de la Asociación de Comerciantes Avenida de la Luz) 
RESUMEN: La Avenida de la Luz, una calle subterránea de Barcelona.-El artículo que se presenta estudia la formación y desarrollo de la Avenida de la Luz, un espacio singular y destacado de Barcelona, tanto por su condición de subterráneo, como por su peculiar evolución histórica y los diferentes usos que ha acogido en su interior. Nació como una galería comercial de lujo en años de penuria y restricción, y a pesar de su deterioro y transformaciones posteriores, ha perdurado como la calle subterránea de la ciudad. El texto reflexiona también sobre el proceso de electrificación en Barcelona, evaluando su destacado impacto socio-cultural y urbanístico. Del mismo modo se realiza una aproximación a los cambios producidos en la estructura comercial de la ciudad, las nuevas formas de comercio, y la creación de nuevos espacios de consumo, desde la apertura de la Avenida de la Luz en 1940 hasta la actualidad.

PALABRAS ClAVE: Proceso de electrificación, galería comercial, calle subterránea.

ABSTRACT: Light Avenue, a subterranian street of Barcelona.-The present article focuses on the begining and development of the Avenida de la Luz. It is a singular and outstanding space of Barcelona, not only for its subterranian character but also for its peculiar historial evolution and the different holded uses. It was born as a luxury commercial gallery in a restriction and poorness period, and despite of its deterioration and later changes, it has last longed as the subterranian street of the city. The report also discusses the electrification process in Barcelona, evaluating its important sociocultural and urbanistic impact. Linked to this, the article talks about the city commercial structure changes, the new trade patterns, and the making of new consume spaces, from the inaguration of the Avenida de la Luz in 1940 to the present.

KEY WORDS: Electrification process, commercial gallery, subterranean street.

REsumÉ: Avenue de la Lumière, une rue souterraine de Barcelone.-L'article présenté étudie la formation et developpement de la Avenida de la Luz, un espace particulier et notable de Barcelona, par sa condition de souterraine, mais aussi du point de vue de sa propre évolution historique et de ses differentes utilisations. Elle est apparue, à une époque de pénurie et de restrictions, comme une galerie commerciale de luxe, et malgré la dégradation et les transformations postérieures, elle demeure la rue souterraine de la ville. Le texte propose également une reflexion sur le processus d'electrification de Barcelone, detachant l'impact remarquable au niveau socio-cultural et urbanistique. De la même façon, cet article effectue une approche concernant les modifications apportées quant à la structure commerciale de la ville, nouvelles formes de commerce, et création de nouveaux espaces de consommation, depuis l'overture de la Avenida de la Luz en 1940 jusqu'à nos jours.

MoTS CLÉ: Processus d'electrification, gallerie commerciale, rue souterraine. 